\title{
EL TESTIMONIO EN LA ENCRUCIJADA ${ }^{1}$
}

\author{
POR \\ JoHN BeverLey \\ University of Pittsburgh
}

\begin{abstract}
“Puede hablar el subalterno?”, pregunta Gayatri Spivak en una intervención ya famosa. ${ }^{2}$ Y responde, contra nuestra inclinación de identificar a lo subalterno precisamente con la oralidad, con esa "voz" individual cuya insistencia ("Me llamo Rigoberta Menchú, y así me nació la conciencia ... ") define al testimonio como género, que no, no como tal, porque "el subalterno es el nombre del sitio que está tan desplazado ... que hacerlo hablar sería como la llegada de Godot en un autobús". ${ }^{3}$ La respuesta es, por supuesto, sólo en parte irónica. Rigoberta Menchú acaba de ganar el premio Nobel de Paz, y Spivak, como discípula de Paul de Man y Jacques Derrida y representante de la desconstrucción literaria en la academia norteamericana, habla a menudo, aunque desde una posición de triple subordinación con respeto a la hegemonía: como mujer; como ciudadana de un país del Tercer Mundo (la India); y como perteneciente a una minoría étnica (es bengalí) de ese país, cuyo sujeto nacional se suele nombrar inexactamente en español "hindú". Pero, como veremos, el problema del testimonio es también uno de representación y representatividad.

La intervención de Spivak estaba destinada a revelar detrás de la buena fe del intelectual solidario o "comprometido" el trazo de una construcción literaria colonial o neocolonial de un "otro" con el cual podemos hablar (o que se presta a hablar con nosotros), suavizando así nuestra angustia ante la realidad de la diferencia (y del antagonismo), y afirmando la normalidad de nuestra situación de recepción. Es el caso del "informante nativo" de la antropología; de los "traductores" indígenas en la Conquista, como la Malinche; de los esclavos "buenos" como Francisco en la novela cubana liberal antiesclavista; del campesino en el costumbrismo bucólico oligárquico (que, en palabras de
\end{abstract}

\footnotetext{
${ }^{1}$ En una versión distinta, este ensayo apareció originalmente como la introducción a La voz del otro: Testimonio, subalternidad y verdad narrativa, John Beverley y Hugo Achugar eds., número especial de la Revista de Critica Literaria Latinoamericana 36 (1992), reeditado como libro bajo el mismo título (Lima-Pittsburgh: Latinoamericana Editores, 1992). Para su reelaboración, agradezco profundamente los aportes de los participantes en el seminario sobre testimonio que realicé en el Departmento de Lenguas y Literaturas Hispánicas de la Universidad de Pittsburgh en 1992.

${ }^{2}$ Gayatri Spivak, "Can the Subaltern Speak?", en Marxism and the Interpretation of Culture, Cary Nelson y Lawrence Grossberg eds. (Urbana: University of Illinois Press, 1988) 271-313.

${ }^{3}$ Gayatri Spivak, "On the Politics of the Subaltern", entrevista con Howard Winant, Socialist Review, 90/3 (1990) 91.
} 
Sergio Ramírez, "vió el campo desde el balcón”); de la representación de la mujer en la literatura latinoamericana en general. No ayuda tampoco que este sujeto venga envuelto ahora en un ropaje de teoría posestructuralista de última moda, porque, como señala Spivak, "las invocaciones contemporáneas de la 'economía libidinal' y del deseo como interés determinante, combinadas con la política utilitaria de los oprimidos (bajo el dominio del capital socializado) 'hablando por sí mismos', restauran la categoría del sujeto soberano dentro de la teoría que más parece cuestionar esa categoría" ("Subaltern" 278).

¿No sería el testimonio simplemente un nuevo capítulo de una vieja historia de las relaciones "literarias" entre opresores y oprimidos, clases dominantes y subordinadas, metrópolis y colonia, centro y periferia, Primery Tercer Mundos? ¿Y su narrador otro sujeto subalterno que nos entrega junto con su sumisión y la plusvalía - lo que deseamos quizás aún más en un sistema global emergente basado en la acumulación y manipulación de información: su "verdad", verdad que, como al comienzo del testimonio de Rigoberta Menchú, es "toda la realidad de un pueblo"? ${ }^{4}$ De allí que Elzbieta Sklodowska, quizás la investigadora más rigurosa del testimonio, concluya recientemente, contra una aseveración de Jorge Narváez, que el testimonio de hecho "no representa una reacción genuina y espontánea del 'sujeto-pueblo multiforme' frente a la condición postcolonial, sino que sigue siendo un discurso de las élites comprometidas a la causa de la democratización". 5

Miremos, sin embargo, la otra cara de la moneda. Como se sabe, durante la época de Reagan y Bush, la nueva derecha norteamericana hizo de Me llamo Rigoberta Menchú, o más exactamente de su versión en inglés, I, Rigoberta Menchu. An Indian Woman in Guatemala, uno de los blancos de su ataque contra el multiculturalismo y lo que llamaba, en una especie de macarthyismo a la inversa, political correctness (el concepto se refiere a la nueva ortodoxia supuestamente impuesta en la universidad norteamericana por profesores marxistas y feministas, la crítica anti-colonialistay anti-racista, la desconstrucción, etcétera). El testimonio de Menchú fue seleccionado por la prestigiosa Universidad de Stanford para uno de los cursos subgraduados obligatorios de introducción a la cultura occidental, después de un debate en que intervino, en contra de la decisión mayoritaria de la facultad, el entonces Secretario de Educación de la administración Reagan, William Bennett. Un ideólogo neoconservador, Dinesh D'Souza, denunció la decisión en un best-seller, Illiberal Education - educación iliberal — y de allí el tópico de Rigoberta Menchú y su testimonio se generalizó en la prensa norteamericana, apareciendo en, entre otros lugares, el Wall Street Journal, Business Week, Newsweek, y los noticieros televisivos. ${ }^{6}$

Dada la ansiedad que los defensores del programa de estudios literarios tradicional sintieron ante la diseminación de un texto como Me llamo Rigoberta Menchú, no debe

\footnotetext{
${ }^{4}$ Rigoberta Menchú, con Elisabeth Burgos Debray, Me llamo Rigoberta Menchú, y así me nació la conciencia (La Habana: Casa de las Américas, 1983): 30.

${ }^{5}$ Elzbieta Sklodowska, "Hacia una tipología del testimonio hispanoamericano", Siglo XX/20th Century 8, 1-2 (1990-91): 113.

${ }^{6}$ Esta campaña, paradójicamente, incrementó el valor internacional de la figura de Menchú como significante cultural, conduciendo en parte a su nombramiento eventual para el premio Nobel de la Paz. Sobre el debate de Stanford, véase Mary Louise Pratt, "Humanities for the Future: Reflections on the Western Culture Debate at Stanford", en The Politics of Liberal Education, Darryl Glass y Barbara Herrnstein Smith eds. (Durham: Duke University Press, 1992).
} 
sorprendernos mucho que su narradora se declara ella misma posmodernista y practicante de lo que se solía llamar un "anti-humanismo teórico". Dice Menchú:

Ya sea por las religiones, ya sea por las reparticiones de tierra, ya sea por las escuelas, ya sea por medio de libros, ya sea por medio de rodeos, de cosas modernas, nos han querido meter otras cosas y quitar lo nuestro (273; el subrayo es mío).

Su testimonio, quizás el texto literario más interesante producido en América Latina en la década de los ochenta, comienza estratégicamente sin embargo con una denuncia no sólo de la cultura del libro sino del sujeto individual interpelado por ella:

Me llamo Rigoberta Menchú. Tengo veintitrés afios. Quisiera dar este testimonio vivo que no he aprendido en un libro y que tampoco he aprendido sola ya que todo esto lo he aprendido con mi pueblo (30).

Contiene una serie de pasajes como el que acabo de citar que problematizan explícitamente el sistema de educación estatal en Guatemala y el alfabetismo como formas de penetración cultural. Y termina con un nuevo rechazo de intelectuales y libros:

Sigo ocultando lo que yo considero que nadie lo sabe, ni siquiera un antropólogo, ni un intelectual, por más que tenga muchos libros, no saben distinguir nuestros secretos. (377)

La genealogía de esta posición remite, por un lado, a un hecho contemporáneo: la complicidad de la universidad - y de la etnografia en particular - en procesos de genocidio o dominación cultural; por otro, a un hecho histórico: la práctica de los conquistadores españoles de separar a los hijos de la aristocracia indígena mesoamericana de sus familias para enseñarles el español y la doctrina cristiana. Walter Mignolo ha observado de esta práctica que

... muestra que el alfabetismo no se produce sin violencia. La violencia, sin embargo, se ubica no en el hecho de que los niños estén secuestrados día y noche, sino en la prohibición de tener conversaciones con sus padres, sobre todo con sus madres. En una sociedad de oralidad primaria, en la cual casi la totalidad del conocimiento era transmitida por la conversación, la preservación del contacto oral contradecía el esfuerzo de enseñar cómo leer y escribir. Prohibiendo conversaciones con la madre significaba, esencialmente, despojar a los niños de la cultura presente en la lengua y preservada y transmitida en el habla. ${ }^{\text {? }}$

Uno de los aspectos más señalados del testimonio es que permite un reto a la pérdida de la autoridad de la oralidad en el contexto de procesos de modernización cultural que privilegian al alfabetismo y la literatura como normas de expresión. Pero sería erróneo pensar por esto que Rigoberta Menchú, o, por extensión, cualquier narrador testimonial,

\footnotetext{
${ }^{7}$ Walter Mignolo, "Literacy and Colonization: The New World Experience", en 1492-1992: Re/ Discovering Colonial Writing, René Jara y Nicholas Spadaccini eds. (Minneapolis: University of Minnesota, 1989): 67 (traducción mía).
} 
esté proponiendo la oralidad como expresión única o auténtica de su propia subalternidad. Parte de su lucha como adolescente con sus padres - lucha que ella pone al centro de su narración, que es entre otras cosas una especie de Bildungsroman-involucra precisamente su deseo de hacerse catequista, proceso que requería primero memorizary después saber leer y comentar pasajes de la Biblia en quiché. Más tarde siente la necesidad como organizadora campesina de aprender otras lenguas indígenas y el español, experiencia que le permite la producción de su testimonio como texto accesible a un público latinoamericano e internacional.

Lejos de ser "inocente" de lo que representa el libro y la literatura en la cultura occidental, es evidente que Menchú se apodera de y utiliza conscientemente la posibilidad de producir (a través de su interlocutora, la antropóloga venezolana Elisabeth Burgos) un texto accesible a un público lector metropolitano. Hace esto, sin embargo, sin sucumbir a la ideología - nuestra ideología - humanista de lo literario, o lo que viene a ser lo mismo, sin abandonar su identidad y función como miembro de su comunidad para hacerse "escritora". Esta estrategia se diferencia de la autobiografía literaria, donde la posibilidad de hacer literatura - escribir la "vida" de uno mismo- equivale precisamente al abandono de una identidad étnica y de clase, la pérdida del Gemeinschaft o comunidad tierna de la juventud en favor de una individualización secularizadora y modernizadora. ${ }^{8}$

El ejemplo contemporáneo más relevante de este tipo de articulación autobiográfica desde una posición subalterna es, en contraste con el testimonio de Menchú, un texto alabado por la nueva derecha norteamericana, entre otras cosas por su crítica de la política del bilingüismo. Me refiero al libro de Richard Rodriguez (sin acento), Hunger of Memory (Hambre de memoria), que narra el medro social de un niño de origen latino en los Estados Unidos - proceso que involucra una pérdida no sólo de su identidad étnica sino de nombre: el narrador había comenzado su vida como Ricardo Rodríguez (con acento), hijo de una familia mexicana de clase obrera de la ciudad de Sacramento en California. ${ }^{9}$

En Hunger of Memory, la capacidad de medro del narrador se debe precisamente a su apropiación de la literatura como discurso de poder, apropiación ejemplificada en la composición del mismo texto autobiográfico, que es en este sentido autorreflexivo. Rodriguez escribe al comienzo de su relato:

Once upon a time, I was a "socially disadvantaged" child. An enchantedly happy child. Mine was a childhood of intense family closeness. And extreme public alienation. Thirty years later I write this book as a middle-class American. Assimilated (3).

El inglés del original es obligatorio aquí porque el narrador rechaza el bilingüismo como norma cultural. Para Rodriguez, el lenguaje público de la autoridad y el poder es el inglés; la ley del padre que impone la castración simbólica obligatoria para la socialización del sujeto es la necesidad de abandonar el lenguaje materno. Su educación y aprendizaje

\footnotetext{
${ }^{8}$ Véase sin embargo la cuidadosa problematización del género autobiográfico en América Latina hecha por Sylvia Molloy en su libro At Face Value (Cambridge: Cambridge University Press, 1990). ${ }^{9}$ Richard Rodriguez, Hunger of Memory. The Education of Richard Rodriguez (Nueva York: David Godine, 1982).
} 
literario - gracias a una beca precisamente a Stanford, llega a estudiar literatura inglesa del renacimiento- es equivalente entonces al paso del orden imaginario al orden simbólico en el famoso esquema lacaniano. Cuando vuelve a su barrio de la universidad para trabajar durante el verano, observa de sus compañeros de trabajo mexicanos:

Their silence is more telling. They lack a public identity. They remain profoundly alien .... I had finally come face to face with los pobres (138-39).

Richard Rodriguez escribe con elocuencia en inglés a un público lector gringo de la necesidad de integración del latino a la cultura dominante de un país que ya, con una población hispanohablante de entre 25 y 30 millones, es el cuarto o quinto del mundo hispánico. Rodriguez siente que puede hablar, en otras palabras, pero no como subalterno, no como Ricardo Rodríguez. Lo que le separa de su propia subalternidad —del silencio taciturno de los pobres (pero ya hemos visto en Rigoberta Menchú que ese silencio es táctico y no un atributo esencial de grupos sociales subalternos) - es la literatura. En este caso, la literatura produce activamente (en vezde "reflejar" o describir) una situación de subalternidad.

La estrategia del narrador testimonial representa una manera distinta de articular una identidad personal, estrategia que no implica en el caso de narradores de origen popular una separación del estamento social del cual proceden (y que sí implica, por contraste, un deshacerse o desatenderse de una posición de privilegio relativo en el caso de sus interlocutores ${ }^{10}$ ). La naturaleza de la relación entre el narrador testimonial y el grupo social a que pertenece no es exactamente transparente, sin embargo. A pesar de esa metonimia textual que equipara en el testimonio historia de vida individual con historia de grupo o comunidad, el narrador testimonial no es lo subalterno como tal, sino más bien algo como un "intelectual orgánico" (para recordar el concepto de Gramsci) del grupo, comunidad o clase subalterna, que habla a (o en contra de) la hegemonía a través de esta metonomia en su nombre y en su lugar.

Como la relación narrador-interlocutor que está implícita en su producción, el testimonio está situado en la intersección de las formas culturales del humanismo burgués, como la literatura y el libro-mercancía, engendradas por y relacionadas con las prácticas del colonialismo y el imperialismo, y esas prácticas culturales subalternas que a menudo constituyen su "contenido" narrativo-descriptivo. Es una variante de lo que se solía llamar "la dialéctica de opresor y oprimido" en esa frase hecha - quizás anticuada ya- de las luchas anticoloniales. Es y no es una forma "auténtica" de cultura subalterna; es y no es "narrativa oral"; es y no es "documental"; es y no es literatura; concuerda y no concuerda con el humanismo ético que manejamos como nuestra ideología profesional; afirma y desconstruye a la vez la categoría del "sujeto" como centro de representación y protagonismo social.

${ }^{10}$ La evolución de la obra de la escritora centroamericana Claribel Alegría ofrece un ejemplo de esto. Alegría abandona la forma del Bildungsroman lírica cuasi-autobiográfica que había cultivado inicialmente - Cenizas de Izalco (1966) - para emprender la reconstrucción testimonial de la vida de una militante del FMLN salvadoreño - No me agarran viva (1983). En cierto sentido, el problema existencial de la heroína de Cenizas - su vacilación entre su origen oligárquico y la realidad social de su país- sólo puede ser resuelta a través de un desplazamiento a otra forma narrativa. 
En particular, Sklodowska ha apuntado que:

\begin{abstract}
... sería ingenuo (en el caso del testimonio) asumir una relación de homología directa entre la historia y el texto. El discurso del testigo no puede ser un reflejo de su experiencia, sino más bien su refracción debida a las vicisitudes de la memoria, su intención, su ideología La intencionalidad y la ideología del autor-editor se sobreponen al texto original, creando más ambigüedades, silencios y lagunas en el proceso de selección, montaje y arreglo del material recopilado conforme a las normas de la forma literaria. Así pues, aunque la forma testimonial emplea varios recursos para ganar en veracidad y autenticidad -entre ellos el punto de vista de la primera persona-testigo- el juego entre ficción e historia aparece inexorablemente como un problema. ${ }^{11}$
\end{abstract}

Es indudablemente una advertencia útil contra un triunfalismo fácil inducido por el "efecto de realidad" que produce la narración en primera persona y la situación de urgencia del testimonio. Revela la complejidad del testimonio, desmistificando así la posibilidad de su recepción "costumbrista" (y neo-colonial) como representación transparente de una voz popular (hecha "para nosotros"). Subraya que la subalternidad es una identidad relacional y no esencial u ontológica. El peligro que conlleva a la vez, sin embargo, es diluir lo que es particular al testimonio como forma - su capacidad de, en palabra de Margaret Randall, "reconstruir la verdad" de lo subalterno e imponer esta reconstrucción como una demanda ética y política al lector. Este desliz equivaldría una recepción "liberal" del testimonio, que permite su otredad y alienta su incorporación al canon, pero a costa de relativizar su poder estético-epistemológico especial, haciendo del testimonio algo sancionado por el poder y la autoridad académica, y de ahí una construcción no-antagónica del sujeto subalterno por la hegemonía. Como hemos visto en las propias palabras de Rigoberta Menchú, el testimonio no quiere ser simplemente "otra" forma de literatura.

El deseo y la posibilidad de producir testimonios, la creciente popularidad del género, indican que hay experiencias vivenciales en el mundo hoy que no pueden ser representadas adecuadamente en las formas tradicionales de la literatura, que en cierto sentido serían traicionadas por éstas. En la negociación entre oralidad y escritura que implica su complicado mecanismo de narración y transcripción textual, el testimonio se sitúa dentro de la problematización posmoderna más amplia de la literatura en sí como una práctica cultural falocéntrica. Emerge en el escenario cultural latinoamericano de las últimas décadas como un reto y una alternativa a la vez a la producción narrativa del Boom. Desplaza, en particular, la centralidad del escritor como héroe cultural —el "conductor de pueblos" del americanismo literario liberal - todavía evidente en esa producción. Pero al mismo tiempo es evidente que el testimonio constituye una nueva forma $\longrightarrow$ género- de literatura, con una institucionalización pedagógica correspondiente. El problema de aculturación negativa sugerido por la autobiografia de Rodríguez - que tiene, al igual que el testimonio de Menchú, a la universidad de Stanford como contexto institucionalrecuerda forzosamente que la incorporación de formas culturales subalternas a la torre de marfil del humanismo acádemico-literario puede esconder a veces una lucha a muerte para

${ }^{11}$ Elzbieta Sklodowska, "La forma testimonial y la novelística de Miguel Barnet", Revista/Review Interamericana XII, 3 (1982) 379. 
el poder de la representación. La tensión entre testimonio y literatura culta, y entre narrador y interlocutor/lector de un testimonio, es una tensión no sólo históricamente determinada sino necesaria en el mundo actual.

El problema de la recepción y utilización pedagógica del testimonio se relaciona con el problema de su veracidad sugerido por Sklodowska. El antropólogo norteamericano David Stoll vivió entre 1988 y 1989 en la región del altiplano guatemalteco de donde viene Rigoberta Menchú, estudiando el proceso de evangelización de las comunidades indígenas por sectas protestantes. Esta experiencia le condujo a cuestionar la representatividad del testimonio de Menchú. Según él, la descripción que ella hace allí de la tortura y el asesinato de su hermano y otros veinte victimas en la plaza del pueblo de Chajúl por el ejército guatemalteco es "una invención literaria" (a literary invention). ${ }^{12}$ Añade que no es una fabricación: de hecho el ejército torturó y mató al hermano de Menchú en, o cerca de, Chajúl. Pero los detalles precisos del incidente que ella ofrece en el texto de Me llamo Rigoberta Menchú no coinciden con los recuerdos de la gente de Chajúl que Stoll entrevistó, ni en general con las crónicas de la muerte del hermano que aparecieron en la documentación de las comisiones de derechos humanos. Según estas fuentes, apunta Stoll, el hermano no fue quemado publicamente ante su familia, como narra Menchú; más bien fue fusilado en otra fecha en las afueras del pueblo.

Se podría alegar que las entrevistas en que se basa Stoll ocurrieron casi diez años después de los incidentes relatados por Menchú, años que presenciaron la derrota del la posibilidad revolucionaria que su testimonio representaba, y por lo tanto un cambio en la memoria histórica de estas comunidades; 0 , en otras palabras, que la violencia represiva produce, según el concepto del antropólogo Michael Taussig, una especie de "lobreguez epistémica" (epistemic murk) ${ }^{13}$ entre los informantes; o que un estudio cuidadoso de la información disponible revelaría fuentes que apoyan la versión de Menchú (el mismo Stoll admite que una de las crónicas de las organizaciones de derechos humanos coincide con la narrativa de Menchú); o, como ha sugerido Doris Sommer, que la misma narradora desconstruye en su discurso la ilusión de que se nos está entregando "toda la realidad" de su pueblo. ${ }^{14}$

Pero, ¿qué pasa si aceptamos simplemente que de hecho se trata de una "invención literaria"? Evidentemente problematiza la distinción entre testimonio y literatura que acabamos de trazar en respuesta a lo apuntado por Sklodowska. Yo había sugerido que las descripciones de tortura y muerte en Me llamo Rigoberta Menchú representaban por su grado de elaboración e intensidad descriptiva una especie de "realismo mágico" testimonial. ${ }^{15}$ Un amigo, el crítico jamaiquino Robert Carr, me ha culpado de estetizar el testimonio

\footnotetext{
${ }^{12}$ David Stoll "I, Rigoberta Menchu and Human Rights reporting in Guatemala". Mimeografia de una ponencia presentada en el congreso sobre "Political Correctness" and Cultural Studies, 20 de octubre 1990, Western Humanities Institute, University of California, Berkeley, 6.

${ }^{13}$ Véase su Shamanism, Colonialism, and the Wild Man. A Study in Terror and Healing (Chicago: University of Chicago Press, 1987), passim.

${ }_{14}$ Doris Sommer, "Rigoberta's Secrets", Latin American Perspectives 70, 18/3 (1991): 32-50; traducción: "Sin secretos", Revista de Crítica Literaria Latinoamericana 36 (1992): 135-153.

15 John Beverley, "The Margin at the Center: On Testimonio", Modern Fiction Studies 35, 1 (1989) 21.
} 
al decir esto. ${ }^{16}$ Puede ser; sin embargo, me parece que el punto no carece totalmente de validez. Menchú no narra simplemente de un modelo "oral" indígena (pero tampoco conocemos las técnicas y géneros de la narración oral maya). Su experiencia como catequista de la Biblia evidentemente afecta su manera de narrar (es la función del catequista dramatizar los acontecimientos que narra para su público). Recordando la distinción aristotélica entre poesía y historia en Poética, el testimonio no es historia en el sentido de una mera aglomeración de particulares; aspira a ser ejemplar en su especificidad. En sus descripciones de tortura y represión, Menchú está tratando de dar una impresión de la fuerza de la violencia que destruyó no sólo a su hermano sino a la mayor parte de su familia. Igualmente, su evocación de la comunidad maya, la cual Stoll encuentra idealizada, es, como cualquier proyecto ideológico, en parte realista, en parte heurística y utópica. (De hecho, por la misma naturaleza del lenguaje o los códigos semióticos, ningún discurso es o puede ser coincidente con lo real; toda narración se desarrolla en parte en un registro imaginario.) $)^{17}$

Sería, creo, otra versión del native informant antropológico conceder al narrador testimonial la posibilidad de ser sólo un testigo verídico, pero no la de crear una narrativa épica propia (cuya construcción permite, según un famoso debate en la preceptiva literaria renacentista, el uso de meraviglia - cosas maravillosas o imaginarias, como el personaje de Orlando- para ilustrar la historia). No es casual que Stoll relacione su cuestionamiento de la representatividad de Me llamo Rigoberta Menchú con un desencanto con lo que él entiende por una antropología "posmoderna": "lo que quiero decir es que si nuestro marco es el texto, la narrativa o la voz en vez de la sociedad, la cultura, o la economía política, es fácil encontrar alguien para decir lo que queremos oír" (11). Pero también Stoll concede que lo único que se podría poner en vez de la narración no adecuadamente representativa de Menchú son ... otros testimonios, testimonios que tampoco pueden ser adecuadamente representativos (en el doble sentido de representación: es decir, mimético y político).

Conocemos ya de sobra la naturaleza del problema: aparte de textos y discursos de varia índole, no hay un nivel de facticidad social que pueda garantizar la veracidad de una u otra representación. La "sociedad" misma no es una esencia previa a su representación discursiva, sino precisamente el resultado de luchas para representary sobre la representación. Esto es el significado del aforismo de Walter Benjamin que Margaret Randall recogió en su

16 "Beverley trata las descripciones de la tortura y la muerte en términos de estilos literarios, argumentando contra la desconstrucción de sus categorías por razones de urgencia política. Al final, parece atrapado entre su deseo de transparencia del sujeto y el hecho de enfrentar palabras en una página, transcritas, editadas y arregladas para circular en un mercado de bienes culturales abierto, cuyo uso en la academia él aboga en aras de una agenda política de la representación ..." Robert Carr, "Re/presentando el testimonio: Notas sobre el cruce divisorio primer mundo/tercer mundo", Revista de Crítica Literaria Latinoamericana 36 (1992) 78.

17 "Any statement of authority has no other guarantee than its very enunciation, and it is pointless for it to seek it in another signifier, which could not appear outside this locus in any way. Which is what I mean when I say that no metalanguage can be spoken, or, more aphoristically, that there is no Other of the Other. And when the Legislator (he who claims to lay down the Law) presents himself to fill the gap, he does so as an impostor". Jacques Lacan, Écrits. A Selection (New York: Norton, 1977) 310-311. 
manual sobre cómo hacer un testimonio:"18 "Aún los muertos no están a salvo"; aun la memoria de los hechos es coyuntural, relativa. El testimonio es un arte de la memoria subalterna.

Con esta aseveración, entramos de frente en el problema de la relación del testimonio con la posmodernidad. Hay una tendencia natural de ver al testimonio como una alternativa tercermundista ante un posmodernismo visto como el "dominante cultural" (Jameson) del Primer Mundo. George Yúdice sugiere una variante de esta idea en su contraste entre el testimonio y la écriture de Joan Didion o Julia Kristeva. Pero no es de sorprender que él crea también que el testimonio, por su naturaleza génerica petite histoire en vez de grand récit, "forma parte de las prácticas culturales que hoy día apuntan al ocaso del "orden de cosas' que corresponde a la modernidad". ${ }^{19}$

Pero, ¿por qué insistir en un concepto tan sujeto a imprecisión $-\mathrm{y}$ tan identificado con la cultura norteamericana- como el posmodernidad? Por dos razones: 1) el concepto de posmodernidad apunta hacia una situación en que, según Jameson en su famoso artículo, "todo en nuestra vida social - desde el valor de cambio y el estado hasta la estructura de la psique - se ha vuelto 'cultural' en una forma todavía no teorizada"; 2) esta fisión de lo cultural, aumentada por el colapso parcial de la distinción entre cultura de élite y cultura de masas, a su vez nace de y corresponde con un desencanto con el proyecto modernizador del estado nacional y una valorización consecuente de la heterogeneidad cultural. De estas condiciones, nace un nuevo sentido de agencia política-cultural, que se diferencia radicalmente de una lógica política tradicional de representación y mobilización vertical (como en el caso de los diversos populismos latinoamericanos). ${ }^{20}$

El testimonio surge precisamente en el contexto de una crisis de representatividad de los viejos partidos políticos, incluidos los de la izquierda. De allí que su correlativo político predilecto sea los llamados "nuevos movimientos sociales", como las Madres de Plaza de Mayo, o el Comité de Unidad Campesina de Menchú, o las comunidades de base de la teología de la liberación: de hecho, todos movimientos que emplean de una manera u otra representaciones testimoniales en su protagonismo (la consigna del grupo norteamericano de activistas del SIDA, ACT-UP, es relevante en este sentido: SILENCE=DEATH (el silencio equivale a la muerte). Yúdice señala al respeto: "Más que representación, estos textos [testimoniales] enfocan las maneras en que diversos grupos oprimidos de mujeres, campesinos, indígenas, trabajadores, domésticas, fieles, squatters, etc. practican su identidad no sólo como resistencia a la opresión sino también como cultura afirmativa, como estética práctica" (Yúdice, 213).

\footnotetext{
${ }^{18}$ Margaret Randall, Testimonios (San José: Centro de Estudios Alforja, 1983).

${ }^{19}$ George Yúdice, "Testimonio y concientización," Revista de Crítica Literaria Latinoamericana 36 (1992): 215.

${ }^{20}$ Norbert Lechner formula la paradoja representada por la experiencia política de la posmodernidad así: "¿Cómo articular una pluralidad de voluntades individuales, en principio ilimitadas, en una voluntad colectiva que, por definición, establece límites? ... (L)a democracia (como principio de legitimidad) presupone una identidad que la democracia (como principio de organización) nunca puede producir como algo permanente y definitivo. "Un desencanto llamado posmoderno", documento de trabajo de la FLACSO (Santiago: FLACSO, s.f.): 7.
} 
Esta aseveración parece limitar el testimonio a las formas de "micro-política" asociadas con estos movimientos o grupos, sin embargo. Pero también existe en la "alegoría nacional" implícita en las relaciones (contradictorias, por supuesto, pero óptimamente del tipo "contradicciones en el seno del pueblo") entre el productor, interlocutor y receptor del testimonio la posibilidad de lo que podriamos llamar un partido o movimiento de izquierda de nuevo tipo, heterogéneo (en el sentido de no reducir las demandas de distintos grupos sociales a una instancia mayor), pero también agrupando sujetos sociales normalmente separados alrededor de un proyecto "nacional" potencialmente hegemónico. Es una posibilidad materializada en distintas formas, por ejemplo, en el partido laborista brasileño, el PDR mejicano de Cárdenas, el Comité de Unidad Campesina de Rigoberta Menchú en Guatemala, la campaña "Vota No" contra la dictadura en Chile, el surgimiento repentino de la organización Causa $\mathrm{R}$ en Venezuela, o (en un contexto no-americano) el Congreso Nacional Africano de Nelson Mandela. Concretamente, tenemos la impresión que, tanto en las revoluciones centroamericanas como en los movimientos civiles en pro de los derechos humanos y la redemocratización en el Cono Sur, el testimonio ha sido no sólo una representación de formas de resistencia y lucha sino también un medio de y hasta un modelo paraéstas. Lo que es evidente en este hecho es que la cuestión de la naturaleza del testimonio es en primera y última instancia política, pero de una política que de una manera "posmoderna" se funda hasta cierto punto en nuevas modalidades estéticas y epistemológicas. ${ }^{21}$

Este hecho, sin embargo, también puede servir para indicar los límites de la efectividad del testimonio. De la misma manera en que hemos visto escasear el poder estéticoideológico de lo que Emir Rodríguez Monegal llamó "las grandes máquinas de narrar" del Boom, la actualidad del testimonio puede ser más y más precaria en un mundo dominado por los medios electrónicos (y nuevas formas narrativas como la telenovela), y por una correspondiente privatización de la experiencia. La narradora testimonial boliviana Domitila Chungara de Barrios expresó al final de su conocido testimonio, Si me permiten hablar, su deseo de ver al texto reintegrado como parte de la cultura de las comunidades mineras cuya historia retrata. De hecho, el testimonio es parte de una "cultura afirmativa" subalterna (aunque dirigido principalmente a un interlocutor "letrado"), donde existen numerosas otras formas de práctica testimonial. Pero, en mi opinión, el testimonio como tal -es decir, como "texto"- puede volver a formar una parte integral de las culturas subalternas que representa paradójicamente sólo a través de experimentar otra alienación:

${ }^{21}$ Sobre este punto, veánse los ensayos reunidos en el número especial de la revista boundary 220 , 3 (1993) sobre The Postmodernism Debate in Latin America, John Beverley y José Miguel Oviedo eds. El manifiesto teórico de la nueva política de "democratización radical" y apertura hacia la heterogeneidad social es Ernesto Laclau y Chantal Mouffe, Hegemony and Socialist Strategy (London: Verso, 1988); sobre los "nuevos movimientos sociales" en particular, véase Laclau, "New Social Movements and the Plurality of the Social", en New Social Movements and the State in Latin America, David Slater ed. (Amsterdam: CEDLA, 1985); y The Making of Social Movements in Latin America. Identity, Strategy, and Democracy, Arturo Escobar y Sonia Alvarez eds. (Boulder: Westview Press, 1992). 
su incorporación a los medios masivos de comunicación. De allí la creciente importancia de documentales o noticieros televisivos en el imaginario cultural americano. ${ }^{22}$

Recordemos, para terminar, un interrogante pre-posmoderno conocido: ¿es preferible el idealismo inteligente al materialismo vulgar? ¿Es preferible una lectura inocente pero solidaria del testimonio a una desconstrucción de su "metafísica de presencia", vista como cómplice con mecanismos neocoloniales de representación y/o con laingenuidad interesada del intelectual burgués o pequeño burgués? La respuesta en ambos casos debe ser que depende de las circunstancias. No es el tipo de lectura en sí del testimonio lo que es válido, sino la manera en que esta lectura se ajusta a las necesidades de lucha (de liberación o simplemente de sobrevivencia) que están involucradas en la situación de enunciación del testimonio. Por el lado de sus interlocutores, el testimonio es evidentemente una manera de "servir al pueblo". Pero, porque se trata de solidaridad - es decir, de un sentido de intereses compartidos - y no de caridad o condescendencia, quizás lo dicho por Rigoberta Menchú sea más exacto como descripción de nuestra propia posición de sujeto ante el testimonio: "Cada uno de nosotros tiene que conocer nuestra realidad y optar por los demás".

\footnotetext{
${ }^{22}$ Hay otro problema relacionado en particular con el texto de Domitila Chungara que incide sobre la actualidad del testimonio. Como señala Javier Sanjinés, la aplicación de una política económica neo-liberal en Bolivia ha conducido en los últimos años a la virtual desaparición de las comunidades mineras y sus modalidades políticas y culturales, y el surgimiento de nuevas formas, "posttestimoniales", si se quiere, de cultura popular. Javier Sanjinés, "Testimonial Discourse and New Popular Trends in Bolivia," Mediations 17, 1 (1992): 50-59.
} 
